\section{Separation of Carotenoids, Steroids and the Related Substances on Lipophilic Sephadex}

\author{
Tetsuya Suzuki and Kiyozo Hasegawa \\ Research Institute for Food Science, \\ Kyoto University, Uji, Kyoto 611 \\ Received November 8, 1973
}

In order to study the biogenesis of carotenoids using radioisotopes in vivo, it is necessary to remove completely steroids from carotenoids. Since both the groups have a common precursor, mevalonic acid, the radioactivity incorporated into steroids may interfere with the precise radioassay for carotenoids. The methods heretofore in use for removing sterols from unsaponifiable material are the digitonide method," the cooling method ${ }^{1 /}$ and thin-layer chromatography. ${ }^{2}$ The present communication deals with the liquid-gel chromatographic separation of carotenes from sterols on Sephadex LH-20.

Phytoene, phytofluene, $\zeta$-carotene, $\alpha$-carotene and lutein were isolated from carrot oil (Nutritional Research Associates, Indiana) by a chromatographic method. ${ }^{31}$ Lutein diacetate was prepared by acetylation of lutein. ${ }^{4}$ Other substances were obtained commercially. $n$-Hexane and petroleum ether were treated with silica gel to remove aromatic hydrocarbons. ${ }^{5}$

Columns of Sephadex LH-20 (Pharmacia Fine Chemicals, Uppsala) were prepared by using the solvent system, chloroform-methanol-n-hexane (65:5:30), and the column size, $1.5 \times 140 \mathrm{~cm}$, unless otherwise stated. Samples were dissolved in a small volume of the same solvent. Elution was carried out at room temperature. Carotenoids were determined spectrophotometrically. ${ }^{6}$. Squalene and squalane were analyzed by gas-liquid chromatography. ${ }^{71}$ Further ${ }^{14} \mathrm{C}$ labelled squalene was also used for analysis. Steroids were analyzed by gas-liquid chromatography, ${ }^{8}$ and by a colorimetric method. ${ }^{9}$ Elution position of each material was also checked by thin-layer chromatography, using silica gel $G$ plate and petroleum etherbenzene $(9: 1)$ and $\mathrm{MgO}-$ Hyflo supercel $(1: 2)$ and petroleum ether-benzene $(95: 5)$ for peak $A$ to $C$, and silica gel $G$ and benzene-ethylacetate $(5: 1)$ for peak $D$ to $G$ (Fig. 1). The elution volumes of the compounds were expressed in terms of RRV, the relative retention volume of the substance to $\beta$-carotene.

Figure 1 shows a part of the results obtained from the experiments performed by using the solvent system, chloroform-methanol-n-hexane $(65: 5: 30)$. In this

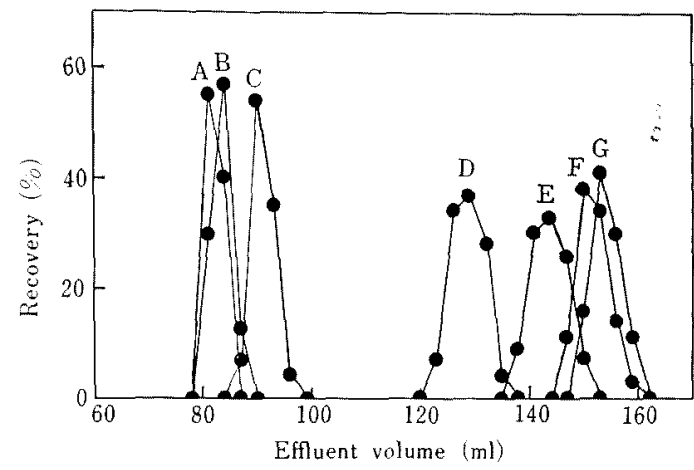

Fig. 1. Separation of Carotenoids, Steroids and the Related Substances on a Sephadex LH-20 Column.

The column used was $1.5 \times 140 \mathrm{~cm}$ and was eluted with chloroform-methanol-n-hexane $(65: 5: 30)$ at a flow rate $6.3 \mathrm{ml} / \mathrm{hr} / \mathrm{cm}^{2}$. Three milliliter fractions were collected.

Phytoene $(50 \mu \mathrm{g})$, phytofluene $(50 \mu \mathrm{g}), \zeta$-carotene $(30 \mu \mathrm{g})$ and lycopene $(50 \mu \mathrm{g})$ were recovered in peak A (RRV, 0.96); $\beta$-carotene $(50 \mu \mathrm{g})$ and $\alpha$-carotene $(50 \mu \mathrm{g})$ in peak B (RRV, 1.00); squalene $(100 \mu \mathrm{g})$ in peak $C$ (RRV, 1.07); lanosterol $(1.0 \mathrm{mg})$ in peak $D$ (RRV, 1.54); $\beta$-sitosterol $(1.0 \mathrm{mg})$ in peak $\mathrm{E}$ (RRV, 1.70); ergosterol $(1.0 \mathrm{mg})$ in peak F (RRV, 1.82); and lutein $(150 \mu \mathrm{g})$ in peak G (RRV, 1.86).

solvent system, carotenes (RRV, $0.96 \sim 1.00$ ) were separated completely free from sterols (RRV, $1.54 \sim$ $1.80)$ and the contamination of squalene (RRV, 1.07) to carotenes was minimum among the solvent systems tested. An increase of column size to $260 \mathrm{~cm}$ caused no better separation than those obtained from $140 \mathrm{~cm}$ column.

Complete separation of carotenes and squalene, a precursor of sterols, was not obtained by the methods generally used, including adsorption chromatography. The present method has an advantage that not only sterols but also squalene can be separated from carotenes. In the cases in which the removal of squalene is not necessary, a short column, $1 \times 10 \mathrm{~cm}$, is sufficient to separate carotenes and sterols.

To investigate the mechanism involved in the separation of the materials, several kinds of compounds, which had different polarities, were also subjected to chromatography. They included lutein diacetate, cholesterylpalmitate, squalane, cholestane, vitamin $\mathbf{K}_{1}$, vitamin A-acetate, vitamin $\mathrm{D}_{2}$ and cholesterol, $\mathrm{RRV}$ values of which were found to be $0.94,0.94,1.06$, $1.06,1.11,1.25,1.60$ and 1.69 , respectively. Since non-polar compounds were eluted before the analogous polar compound, e.g. cholestane (RRV, 1.06) to cholesterol (RRV, 1.69), and carotenes (RRV, 1.00) to lutein (RRV, 1.86), it was suggested that the separation of the compounds on the column was governed mainly by the mechanism of a kind of partition 
chromatography. Contribution of gel filtration mechanism was not so large as seen in comparison of the elution order of cholesterypalmitate (RRV, 0.94) and cholestane (RRV, 1.06) or lutein diacetate (RRV, $0.96)$ and $\beta$-carotene ( RRV, 1.00). The difference in the elution volumes of vitamin $D_{2}(R R V, 1.60)$ and ergosterol (RRV, 1.82) suggested that the difference of the structure in the part of molecule also participate on the separation. The results described above were in accordance with those concerning the steroid separation on lipophilic Sephadex by Eneroth et al. ${ }^{10}$

In the cases in which methanol is used as a main solvent, e.g. methanol-petroleum ether (7:3) and methanol-acetone-petroleum ether $(7: 1: 2)$, the complete separation of carotenes from sterols was not obtained. Furthermore, these solvent systems were unsuitable for the purpose of the experiment because of the poor solubility of carotenes in the solvents.

In conclusion, it was found that Sephadex LH-20 chromatography with non-polar solvent systems such as chloroform-methanol- $n$-hexane was one of the convenient methods to separate sterols from carotenes. The method will greatly aid in obtaining the sterol-free carotene preparation from various biological materials.

\section{REFERENCES}

1) E. I. Mercer, B. H. Davies and T. W. Goodwin, Biochem. J., 87, 317 (1963).

2) L. K. Lowry and C. O. Chichester, Phytochem., 6, 367 (1967).

3) K. L. Simpson, T. O. M. Nakayama and C. O. Chichester, J. Bacteriol., 88, 1688 (1964).

4) P. Karrer and S. Ishikawa, Helv. chim. Acta, 13, 709 (1930).

5) M. M. Graff, R. T. O'Connor and E. L. Skau, Ind. Eng. Chem. Anal. Ed., 16, 556 (1944).

6) "Chemistry and Biochemistry of Plant Pigments," ed. by T. W. Goodwin, Academic Press, London and New York, 1965, pp. $489 \sim 532$.

7) D. G. Anderson and J. W. Porter, Arch. Biochem. Biophys., 97, 509 (1962).

8) W. J. A. Van den Heuvel, J. Sjövall and E. C. Horning, Biochim. Biophys. Acta, 48, 596 (1961).

9) N. Papadopoulos, W. Cevallos and W. C. Hess, J. Neurochem., 4, 223 (1959).

10) P. Eneroth and E. Nyström, Biochim. Biophys. Acta, 144, 149 (1967). 